\title{
The Lattice Structure of Multiplicative Congruential Pseudo-Random Vectors*
}

\author{
By W. A. Beyer, R. B. Roof and Dorothy Williamson
}

\begin{abstract}
The lattice structure of points in an $n$-dimensional space produced by an appropriate grouping of pseudo-random numbers obtained from multiplicative congruential generators is discussed. Examples are given for $2 \leqq n \leqq 6$. The work is based on the theory of the reduction of positive quadratic forms in $n$ variables.
\end{abstract}

1. Introduction. There have recently appeared several articles [3], [8], [13] discussing the distribution of points in an $n$-dimensional Euclidean space $E^{n}$ obtained from multiplicative congruential pseudo-random generators. For example, if $x_{0}, \lambda, \beta$, $\mu$, and $n$ are given positive integers and if

$$
x_{i} \equiv \lambda x_{i-1}\left(\bmod 2^{\beta}\right) \quad(i=1,2, \cdots)
$$

or

$$
x_{i} \equiv \lambda x_{i-1}+\mu\left(\bmod 2^{\beta}\right) \quad(i=1,2, \cdots),
$$

Marsaglia [8] has shown that the points

$$
\left(x_{0}, x_{1}, \cdots, x_{n-1}\right),\left(x_{n}, \cdots, x_{2 n-1}\right), \cdots
$$

may lie on relatively few hyperplanes in $E^{n}$. This idea goes back at least to Franklin [4], [5]. Wood [14] describes a method he used to show that the points actually form a simple lattice in the case $n=2$. Because his methods and results may be of some interest, it was thought that a report giving further details would be appropriate. In addition, a procedure for an extension to $3 \leqq n \leqq 6$ is given. Examples are presented for $2 \leqq n \leqq 6$. A more complete discussion of the general theory is given.

The interest in the present work arises from the need to know whether the generator produces points (3) which lie on few hyperplanes or lie on many. In the first instance, the pseudo-random points will not be uniformly distributed through the hypercube and hence the generator is probably not "good."

While this discussion bears some similarity to that of Coveyou and MacPherson [3], it has some advantages. First, it exhibits precisely the structure of the sets defined by (3). Secondly, it avoids a discussion of the Fourier analysis of lattice structure in which Coveyou and MacPherson couch their work. On the other hand, the present analysis has not been extended beyond $n=6$ (but it is possible to extend the analysis), while Coveyou and MacPherson discuss $2 \leqq n \leqq 10$.

Received June 5, 1970.

AMS 1969 subject classifications. Primary 6515, 1061, 1063.

Key words and phrases. Multiplicative congruential pseudo-random numbers, Lehmer pseudorandom numbers, Monte Carlo integration, random numbers, lattices of random points, testing of random numbers, reduced cells, positive quadratic forms, quadratic forms.

* Work performed under the auspices of the U. S. Atomic Energy Commission.

Copyright @ 1971, American Mathematical Society 
In Section 8 the relation between the discrepancy theory of Zaremba (and others) and the present theory is discussed.

Applications of pseudo-random numbers in Monte Carlo calculations are well known. Applications in digital communications, especially in space communications, may not be so well known. See [6].

The computations were done on the Maniac II computer of the Los Alamos Scientific Laboratory. The Madcap language was used for the coding. This language can readily process arbitrarily large integers which is a requirement of our computations.

2. A Lattice in $E^{n}$. A lattice $G_{n}$ in $E^{n}$ consists of all vectors of the form $\mathrm{y}=$ $\mathbf{e}_{0}+\sum_{i=1}^{n} \mathbf{e}_{i} y_{i}$ where the $\mathbf{e}_{i}(1 \leqq i \leqq n)$ are $n$ fixed linearly independent vectors, the $y_{i}$ are integers, positive, negative, or zero, and $\mathrm{e}_{0}$ is a fixed vector. (This definition is not standard in that the origin is not required to be in the lattice.) The $\left\{e_{i}\right\}$ are said to form a basis of $G_{n}$. Put in other terms, a lattice $G_{n}$ is a coset of a discrete subgroup $H$ of the additive group of vectors in $E^{n}$ where $H$ has $n$ linearly independent vectors. $H$ is discrete if every $x \in H$ has a neighborhood free of points of $H$ other than $x$. The basis vectors of $G_{n}$ are then called generators of $H$.

Following van der Waerden [12, p. 276] one says the basis $\left\{\mathbf{e}_{\boldsymbol{i}}\right\}$ is reduced (in the sense of Minkowski) if:

(1) $\mathbf{e}_{1}$ is the shortest (in the Euclidean norm) of all vectors $\sum_{i=1}^{n} \mathbf{e}_{i} y_{i}$ with the greatest common divisor of $y_{1}, y_{2}, \cdots, y_{n}:\left(y_{1}, \cdots, y_{n}\right)$, equal to 1 ,

(2) $\mathbf{e}_{k}$ is the shortest of all vectors $\sum_{i=1}^{n} \mathrm{e}_{i} y_{i}$ with $\left(y_{k}, \cdots, y_{n}\right)=1$, for $k=2,3, \cdots, n$.

Let $N_{1}$ be the length of the shortest nonzero vector $\mathrm{S}_{1}=\sum_{i=1}^{n} \mathrm{e}_{i} y_{i}$. Let $N_{2}$ be the length of the shortest vector $\mathbf{S}_{2}=\sum_{i=1}^{n} \mathbf{e}_{i} y_{i}$ which is linearly independent of $\mathrm{S}_{1}$. And so on, one defines the successive minima $N_{1}, N_{2}, \cdots$. Then if the $\left\{\mathbf{e}_{i}\right\}$ are reduced, it was shown by Mahler and Weyl [12] that

$$
\left|\mathrm{e}_{i}\right| \leqq \delta_{i} N_{i}, \quad i=1,2, \cdots, n,
$$

where $\delta_{1}=1, \delta_{k}=\max \left(1, \frac{1}{4} \delta_{1}+\frac{1}{4} \delta_{2}+\cdots+\frac{1}{4} \delta_{k-1}+\frac{1}{4}\right)$ for $k=2, \cdots, n$ and that

$$
\left|\mathrm{e}_{i}\right|=N_{i}, \quad i=1,2,3,4,
$$

where $|\mathbf{e}|$ denotes the Euclidean norm. It is this result which connects the reduced bases with the more intuitive idea of the "size" of the fundamental "cell" in the lattice and makes our theory a tool to study the distribution of pseudo-random points in the $n$-cube.

Minkowski [9] has stated the following for $n \leqq 6 .\left(e_{i}\right)_{1 \leqq i \leqq n}$ is reduced if for every subset of $\left(\mathrm{e}_{i}\right)_{1 \leqq i \leqq n}$, say $\left(\mathrm{e}_{i_{j}}\right)_{1 \leq i \leq k}$, one has

$$
\left|\mathrm{e}_{i_{j}}\right| \leqq\left|\sum_{l=1}^{k}( \pm) C_{l} \mathrm{e}_{i_{l}}\right|, \quad j=1,2, \cdots, k,
$$

for all combinations of \pm signs and $\left(C_{l}\right)_{1 \leqq l \leqq k}$ ranging over the following values. If $k=2,3$, and $4, C_{l}=1$. For $k=5$, one of the $C_{l}$ takes the values 1 and 2 and the remainder take the value 1 . For $k=6$, one of the $C_{l}$ takes the values $1,2,3$, another $C_{l}$ takes the values 1 and 2 , and the remainder take the value 1 . (The cases $k=5$, 6 are stated [9] without proof.) 
The analysis given in van der Waerden [12] can be used to obtain algorithms for $n>6$, but would not be optimal algorithms as is so for $2 \leqq n \leqq 6$.

For a set of vectors in $E^{n}:\left(a_{i}\right)_{1 \leqq i \leqq n}$, define det $\left(a_{i}\right)$ to be the $n \times n$ determinant whose $i$ th row consists of the components of the vector $a_{i}$. It is easy to show (see Cassels [2, p. 11, lines 7 to 13]) that for a given lattice $G_{n}$, a set $\left(a_{i}\right)_{0 \leqq i \leqq n}$ in $G_{n}$ defines a basis $\left(a_{i}-a_{0}\right)_{1 \leqq i \leqq n}$ of $G_{n}$ if and only if $0<\left|\operatorname{det}\left(a_{i}-a_{0}\right)\right| \leqq\left|\operatorname{det}\left(a_{i}^{\prime}-a_{0}^{\prime}\right)\right|$ for any any other set $\left(a_{i}^{\prime}\right)_{0 \leqq i \leqq n}$ in $G_{n}$ such that det $\left(a_{i}^{\prime}-a_{0}^{\prime}\right) \neq 0$. Further (see Cassels [2, pp. 9 and 10]), two sets $\left(a_{i}\right)_{0 \leqq i \leqq n}$ and $\left(a_{i}^{\prime}\right)_{0 \leqq i \leq n}$ in $G_{n}$ both define a basis of $G_{n}$ if and only if there exists an $n \times n$ matrix $T$ with integer entries and with det $T= \pm 1$ (unimodular matrix) so that $\left[a_{i}^{\prime}-a_{0}^{\prime}\right]=T\left[a_{i}-a_{0}\right]$, where $\left[a_{i}-a_{0}\right]$ is the $n \times n$ matrix whose $i$ th row is the vector $a_{i}-a_{0}$.

A reduction algorithm is a procedure for obtaining from a basis of a lattice a reduced basis. Reduction algorithms are described and applied for $2 \leqq n \leqq 6$.

3. The Lattice Structure of Multiplicative Congruential Pseudo-Random Vectors. In this section $n$ is an arbitrary positive integer. The following Lemmas 1 and 2 will be needed. They are taken from the book of Jansson [7, p. 68].

LEMMA 1. (1) When $\lambda \equiv 3(\bmod 8)$ and $x_{0} \equiv 1$ or $3(\bmod 8)$, each sequence produced by (1) is some permutation of all the numbers $8 \nu+1$ and $8 \nu+3(\nu=0$, $\left.1, \cdots, 2^{\beta-3}-1\right)$.

(2) When $\lambda \equiv 3(\bmod 8)$ and $x_{0} \equiv 5$ or $7(\bmod 8)$, each sequence produced by $(1)$ is some permutation of all the numbers $8 \nu+5$ and $8 \nu+7\left(\nu=0,1, \cdots, 2^{\beta-3}-1\right)$.

(3) When $\lambda \equiv 5(\bmod 8)$ and $x_{0} \equiv 1(\bmod 4)$, each sequence produced by $(1)$ is some permutation of all the numbers $4 \nu+1\left(\nu=0,1, \cdots, 2^{\beta-2}-1\right)$.

(4) When $\lambda \equiv 5(\bmod 8)$ and $x_{0} \equiv 3(\bmod 4)$, each sequence produced by $(1)$ is some permutation of all the numbers $4 \nu+3\left(\nu=0,1,2, \cdots, 2^{\beta-2}-1\right)$.

Remark. A method of determining exactly what permutation occurs is illustrated by the following discussion.

Consider the case $\lambda \equiv 5(\bmod 8)$ and $x_{0}=1$. Denote the sequence generated by $\lambda=5$ by

$$
S_{0}=\left\{x_{i}^{(0)} ; i=0,1,2, \cdots, 2^{\beta-2}-1\right\}
$$

with $x_{i}^{(0)} \equiv 5^{i}\left(\bmod 2^{\beta}\right)$. So every multiplier $\lambda \equiv 5(\bmod 8)$ with $0<\lambda<2^{\beta}$ occurs among the odd members of $S_{0}$. Every $\lambda \equiv 5(\bmod 8), 0<\lambda<2^{\beta}$, multiplier has a representation of the form $\lambda=x_{2 n+1}^{(0)} \equiv 5^{2 n+1}\left(\bmod 2^{\beta}\right)$. Let $S_{n}$ be the sequence generated with $\lambda=x_{2 n+1}^{(0)}: S_{n}=\left\{x_{i}^{(n)} ; i=0,1, \cdots, 2^{\beta-2}-1\right\}$. One has

$$
\begin{aligned}
x_{i}^{(n)} & \equiv\left[x_{2 n+1}^{(0)}\right]^{i}\left(\bmod 2^{\beta}\right)\left[5^{2 n+1}\right]^{i}=\left(\bmod 2^{\beta}\right) \\
& =5^{(2 n+1) i}\left(\bmod 2^{\beta}\right)=x_{(2 n+1) i}^{(0)} ;
\end{aligned}
$$

i.e. when the multiplier $\lambda \equiv x_{2 n+1}^{(0)}$ is used, the sequence obtained from $x_{i+1} \equiv$ $\lambda x_{i}\left(\bmod 2^{\beta}\right), x_{0}=1$, consists of selecting every $(2 n+1)$ th number from (4), beginning with the first.

LEMMA 2. If, in $(2), \lambda \equiv 1(\bmod 4)$ and $\mu \equiv 1(\bmod 2)$, then $(2)$ produces a permutation of the numbers $0,1,2, \cdots, 2^{\beta}-1$.

The following lemma is needed in the subsequent development.

Lemma 3. Let $A \subset E^{n}$ be a point set such that every point in $A$ has integer co- 
ordinates and there are $n+1$ vectors in $A$, say $\mathbf{e}_{i}, 0 \leqq i \leqq n$, so that $\mathbf{e}_{i}-\mathbf{e}_{0}, 1 \leqq i \leqq n$, are linearly independent. Suppose for any $\mathrm{a}_{i} \in A, 0 \leqq i \leqq n$, and any set of integers $k_{i}$, $1 \leqq i \leqq n$, it is so that $\mathrm{a}_{0}+\sum_{i=1}^{n} k_{i}\left(\mathrm{a}_{i}-\mathrm{a}_{0}\right) \in A$. Then $A$ is a lattice.

Proof. Choose $\mathrm{e}_{i}, 0 \leqq j \leqq n$, in $A$ so that $\left|\operatorname{det}\left(\mathrm{e}_{i}-\mathrm{e}_{0}\right)\right|=D$ has the least positive value where $1 \leqq i \leqq n$. By the hypothesis, every $\mathrm{e}_{0}+\sum_{i=1}^{n} k_{i}\left(\mathrm{e}_{i}-\mathrm{e}_{0}\right) \in A$ where the $k_{i}$ are arbitrary integers. Let a be an arbitrary vector in $A$. Since $\left\{\mathrm{e}_{i}-\mathrm{e}_{0} ; i=1,2, \cdots, n\right\}$ is a linearly independent set, there exist real $\gamma_{i}$ such that $\mathbf{a}-\mathbf{e}_{0}=\sum_{i=1}^{n} \gamma_{i}\left(\mathbf{e}_{i}-\mathbf{e}_{0}\right)$. Suppose $\gamma_{i}$ is not an integer for some $j, 1 \leqq j \leqq n$. Form

$$
\begin{array}{|c}
\left|\begin{array}{c}
\mathbf{e}_{1}-\mathbf{e}_{0} \\
\vdots \\
\mathbf{e}_{i-1}-\mathbf{e}_{0} \\
\mathbf{e}_{i+1}-\mathbf{e}_{0}-\left[\gamma_{i}\right]\left(\mathbf{e}_{i}-\mathbf{e}_{0}\right)-\mathbf{e}_{0} \\
\vdots \\
\mathbf{e}_{n}-\mathbf{e}_{0}
\end{array}\right|=\left|\begin{array}{c}
\mathbf{e}_{1}-\mathbf{e}_{0} \\
\vdots \\
\mathbf{e}_{i-1}-\mathbf{e}_{0} \\
\vdots \sum_{i=1}^{n}\left(\gamma_{i}-\delta_{i j}\left[\gamma_{i}\right]\right)\left(\mathbf{e}_{i}-\mathbf{e}_{0}\right) \\
\mathbf{e}_{i+1}-\mathbf{e}_{0} \\
\vdots \\
\mathbf{e}_{n}-\mathbf{e}_{0} \\
\mathbf{e}_{1}-\mathbf{e}_{0} \\
\vdots \\
\mathbf{e}_{i-1}-\mathbf{e}_{0} \\
\left(\gamma_{i}-\left[\gamma_{j}\right]\right)\left(\mathbf{e}_{i}-\mathbf{e}_{0}\right) \\
\mathbf{e}_{i+1}-\mathbf{e}_{0} \\
\vdots \\
\mathbf{e}_{n}-\mathbf{e}_{0}
\end{array}\right|=\left|\gamma_{i}-\left[\gamma_{j}\right]\right| D
\end{array}
$$

where $\left[\gamma_{i}\right]$ denotes largest integer in $\gamma_{j}$. Since $0<\left|\gamma_{i}-\left[\gamma_{i}\right]\right| D<D$, it is false that $D$ is the minimum positive value of $\left|\operatorname{det}\left(\mathrm{e}_{i}-\mathrm{e}_{0}\right)\right|$ where $\mathrm{e}_{i} \in A$. Thus the $\gamma_{i}$ are integers and therefore every $\mathrm{a} \in A$ has a representation $\mathrm{a}=\mathrm{e}_{0}+\sum_{i=1}^{n} \gamma_{i}\left(\mathrm{e}_{i}-\mathrm{e}_{0}\right)$ where the $\gamma_{i}$ are integers. This completes the proof of the lemma. For a more general lemma, see Cassels [2, p. 78].

In Lemmas 4 to 8 the points defined by (1) or (2) and (3) or (1) or (2) and

$$
\left(x_{0}, x_{1}, \cdots, x_{n-1}\right),\left(x_{1}, \cdots, x_{n}\right),\left(x_{2}, \cdots, x_{n+1}\right), \cdots
$$

are discussed. We make the following convention: The point sets (3) and (5) are to be regarded as point sets $G_{n}$ in $E^{n}$ which are continued by periodicity throughout $E^{n}$; i.e., if $\left(t_{1}, t_{2}, \cdots, t_{n}\right) \in G_{n}$, then $\left(t_{1}+h_{1} 2^{\beta}, t_{2}+h_{2} 2^{\beta}, \cdots, t_{n}+h_{n} 2^{\beta}\right) \in G_{n}$ for all positive, zero, and negative integers $h_{i}$.

Remark. It might be objected that points generated by (1) and (5) or (2) and (5) would make a poor random-point generator, since such points would be highly correlated over a short run. However, the points defined by (1) and (3) or (2) and (3) 
are a reasonably sized subset for small $n$ of those mentioned before and a discussion of the lattice structure of (5) gives information about the lattice structure of (3).

LEMMA 4. If in $(1) \lambda \equiv 5(\bmod 8)$ and $x_{0} \equiv 1(\bmod 4)$, then the point set $G_{n}$ given by (5) forms a lattice in $E^{n}$.

Proof. If $\mathrm{u}_{i}, 1 \leqq i \leqq n$, are the unit coordinate vectors in $E^{n}$ and $\mathrm{x} \in G_{n}$, the vectors $\mathbf{x}, \mathbf{x}+2^{\beta} \mathbf{u}_{i}$ are $n+1$ vectors in $G_{n}$ for which $\left(\mathbf{x}+2^{\beta} \mathbf{u}_{i}\right)-\mathbf{x}$ are linearly independent. Let $\mathbf{x}_{i}, 0 \leqq j \leqq n$, be $n+1$ points of $G_{n}$, not necessarily distinct. Let $k_{i}$, $1 \leqq i \leqq n$, be arbitrary integers. Recall that $x_{i_{j}}=\left(x_{i_{i}}, x_{i_{j}+1}, \cdots, x_{i_{j}+n}\right)=\left(x_{i_{i}}, \lambda x_{i_{j}}+\right.$ $\left.h_{1} 2^{\beta}, \lambda^{2} x_{i_{i}}+h_{2} 2^{\beta}, \cdots, \lambda^{n-1} x_{i_{i}}+h_{n-1} 2^{\beta}\right)$ for some integers $h_{i}$ where $x_{i_{i}}$ is the $i_{j}$ th number generated by (1). Form

$$
\begin{aligned}
& \mathbf{x}= \mathbf{x}_{i_{0}}+\sum_{j=1}^{n} k_{i}\left(\mathbf{x}_{i_{i}}-\mathbf{x}_{i_{0}}\right) \\
&=\left\{x_{i_{0}}+\sum_{i=1}^{n} k_{j}\left(x_{i_{j}}-x_{i_{0}}\right), \lambda\left[x_{i_{0}}+\sum_{j=1}^{n} k_{j}\left(x_{i_{i}}-x_{i_{0}}\right)\right]+h_{1} 2^{\beta}, \cdots,\right. \\
&\left.\lambda^{n-1}\left[x_{i_{0}}+\sum_{j=1}^{n} k_{i}\left(x_{i_{i}}-x_{i_{0}}\right)\right]+h_{n-1} 2^{\beta}\right\}
\end{aligned}
$$

where $h_{i}$ are again integers and the $k_{i}$ are arbitrary integers. Since every $x$ in the sequence generated by (1) under the hypothesis on $\lambda$ and $x_{0}$ has the form $4 \nu+1$, $\nu=0,1, \cdots, 2^{\beta-2}-1$ (Lemma 1, Part 3), $x_{i_{0}}+\sum_{i=1}^{n} k_{i}\left(x_{i_{i}}-x_{i_{0}}\right)$ has the form $4 \nu+1$ for some integer $\nu$. But every number of this form can be expressed as $4 \nu+1=$ $4 \nu_{1}+1+h 2^{\beta}$ with $0 \leqq \nu_{1} \leqq 2^{\beta-2}-1$ and $h, \nu_{1}$ as integers. Thus $\mathrm{x} \in G_{n}$. Lemma 3 can now be applied to give the conclusion of the theorem.

In a similar way, Lemmas 5 to 8 can be proved, using Lemmas 1, 2, and 3 .

LEMMA 5. If, in $(1), \lambda \equiv 5(\bmod 8)$ and $x_{0} \equiv 3(\bmod 4)$, then the point set $G_{n}$ given by (5) forms a lattice in $E^{n}$.

LEMMA 6. In $(1)$, let $\lambda \equiv 3(\bmod 8)$ or $\lambda \equiv 5(\bmod 8)$ and let $x_{0}$ be odd. Then the set $\left(x_{2 n}, x_{2 n+1}\right), n=0,1,2, \cdots$, is a lattice in $E^{2}$.

LEMMA 7. In (1), let $\lambda \equiv 3(\bmod 8)$. Let $G_{n}$ be the set of points in $(5)$ determined with $x_{0} \equiv 1$, or $3(\bmod 8)$ and $G_{n}^{\prime}$ be the same set, but with $x_{0} \equiv 5$ or $7(\bmod 8)$. Then $G_{n} \cup G_{n}^{\prime}$ forms a lattice in $E^{n}$.

LEMMA 8. In (2), let $\lambda \equiv 1(\bmod 4)$ and $\mu \equiv 1(\bmod 2)$. Then the set of points in (5) form a lattice in $E^{n}$ and the basis vectors of the lattice do not depend on $\mu$. The sequence $\left(x_{2 n}, x_{2 n+1}\right), n=0,1,2, \cdots$, forms a lattice in $E^{2}$.

Remark 1. The points $x_{i}$ (extended by our convention) defined by

$$
x_{i} \equiv 3 x_{i-1}\left(\bmod 2^{3}\right), \quad x_{0}=1,
$$

do not form a lattice on the line.

Remark 2. The structure of sequences generated by other generators, such as

1. $x_{n+1} \equiv \lambda x_{n}+\mu\left(\bmod p^{\beta}\right)(p$ an odd prime),

2. $x_{n+1} \equiv \lambda x_{n}\left(\bmod 10^{\beta}\right)$,

3. $x_{n+1} \equiv a_{0} x_{n}+a_{1} x_{n-1}+\cdots+a_{j} x_{n-i}(\bmod p)(p$ a prime),

4. $x_{n+1} \equiv x_{n}+x_{n-1}\left(\bmod 2^{\beta}\right)$,

is discussed in Jansson [7] and a theory analogous to that discussed here might be developable. 
4. Reduction Algorithm in the Case $n=2$. Let $G_{2}$ be a lattice with a basis $\left(\mathbf{e}_{1}, \mathbf{e}_{2}\right)$. Then, by the discussion in Section 2 , if $w$ is an integer, $\left(\mathbf{e}_{1}, \mathbf{e}_{2}+w \mathbf{e}_{1}\right)$ is a basis of $G_{2}$ since

$$
\left(\begin{array}{c}
\mathbf{e}_{1} \\
\mathrm{e}_{2}+w \mathrm{e}_{1}
\end{array}\right)=\left(\begin{array}{ll}
1 & 0 \\
w & 1
\end{array}\right)\left(\begin{array}{l}
\mathbf{e}_{1} \\
\mathbf{e}_{2}
\end{array}\right)
$$

and the matrix $\left(\begin{array}{cc}1 & 0 \\ w & 1\end{array}\right)$ is unimodular. $w$ is chosen to minimize $\left(\mathrm{e}_{2}+w \mathbf{e}_{1}\right)^{2}$. Hence $w$ must satisfy $\left(\mathrm{e}_{2}+(w-1) \mathrm{e}_{1}\right)^{2} \geqq\left(\mathrm{e}_{2}+w \mathrm{e}_{1}\right)^{2} \leqq\left(\mathrm{e}_{2}+(w+1) \mathrm{e}_{1}\right)^{2}$ or

$$
-\frac{\mathrm{e}_{1} \mathrm{e}_{2}}{\mathrm{e}_{1}^{2}}-\frac{1}{2} \leqq w \leqq-\frac{\mathrm{e}_{1} \cdot \mathrm{e}_{2}}{\mathrm{e}_{1}^{2}}+\frac{1}{2}
$$

In order to determine $w$ uniquely, the right-hand inequality in (6) is replaced by $<$ to give

$$
-\frac{\mathrm{e}_{1} \cdot \mathrm{e}_{2}}{\mathrm{e}_{1}^{2}}-\frac{1}{2} \leqq w<-\frac{\mathrm{e}_{1} \cdot \mathrm{e}_{2}}{\mathrm{e}_{1}^{2}}+\frac{1}{2}
$$

Call the basis $\left(\mathrm{e}_{1}, \mathrm{e}_{2}+w \mathrm{e}_{1}\right)$ thus determined $\left(\mathrm{e}_{1}, \mathrm{e}_{2}^{\prime}\right)$. Replace the basis $\left(\mathrm{e}_{1}, \mathrm{e}_{2}^{\prime}\right)$ by a new basis $\left(\mathbf{e}_{1}+w^{\prime} \mathbf{e}_{2}^{\prime}, \mathbf{e}_{2}^{\prime}\right)$ where $w^{\prime}$ is the unique integer determined by

$$
-\frac{\mathbf{e}_{1} \cdot \mathbf{e}_{2}^{\prime}}{\mathbf{e}_{2}^{\prime 2}}-\frac{1}{2} \leqq w^{\prime}<-\frac{\mathbf{e}_{1} \cdot \mathbf{e}_{2}^{\prime}}{\mathbf{e}_{2}^{\prime 2}}+\frac{1}{2} \text {. }
$$

The above procedure is then iterated until two successive minimizing integers of the form $w$ and $w^{\prime}$ are zero. The resulting basis $\left(\overline{\mathrm{e}}_{1}, \overline{\mathrm{e}}_{2}\right)$ is reduced since, from (7), $\overline{\mathrm{e}}_{1}^{2} \geqq$ $2 \overline{\mathbf{e}}_{1} \cdot \overline{\mathbf{e}}_{2} \geqq-\overline{\mathbf{e}}_{1}^{2}$ and, from (8), $\overline{\mathbf{e}}_{2}^{2} \geqq 2 \overline{\mathbf{e}}_{1} \cdot \overline{\mathbf{e}}_{2} \geqq-\overline{\mathbf{e}}_{2}^{2}$ and therefore

$$
\overline{\mathrm{e}}_{1}^{2} \geqq 2\left|\overline{\mathrm{e}}_{1} \cdot \overline{\mathrm{e}}_{2}^{\prime}\right| \text { and } \overline{\mathrm{e}}_{2}^{2} \geqq 2\left|\overline{\mathrm{e}}_{1} \cdot \overline{\mathrm{e}}_{2}\right|
$$

which implies that $\overline{\mathbf{e}}_{1}$ and $\overline{\mathbf{e}}_{2}$ are in length less than or equal to the length of the diagonals of the parallelograms which have $\overline{\mathbf{e}}_{1}, \overline{\mathbf{e}}_{2}$ as adjacent sides. The above algorithm must eventually terminate since for each pair of steps of the algorithm for which $w$ and $w^{\prime}$ is not both zero, the vectors $\left(\mathrm{e}_{1}, \mathbf{e}_{2}\right)$ with integer coordinates are replaced by a pair of vectors $\left(\mathbf{e}_{1}^{\prime}, \mathbf{e}_{2}^{\prime}\right)$ with integer coordinates such that $\left|\mathbf{e}_{1}^{\prime}\right| \leqq\left|\mathbf{e}_{1}\right|$ and $\left|\mathbf{e}_{2}^{\prime}\right| \leqq\left|\mathbf{e}_{2}\right|$, with strict inequality in one of the cases.

5. Reduction Algorithm in the Case $3 \leqq n \leqq 6$. Assume $3 \leqq n \leqq 6$. Let $E=$ $\left(\mathrm{e}_{1}, \mathrm{e}_{2}, \cdots, \mathrm{e}_{n}\right)$ be a set of basis vectors of a lattice $G_{n}$. Stage 1 of the reduction algorithm consists in successively replacing each pair of distinct vectors in $E$ by a reduced pair, using the reduction algorithm for $n=2$. This replacement defines a unimodular transformation from $E$ to new set of vectors $E^{\prime}$ and hence $E^{\prime}$ is a basis of $G_{n}$. This operation is repeated until no further reduction by pairs is possible.

Stage 2 of the algorithm consists in examining for each $k$-tuple $\left(e_{i_{j}}\right)_{1 \leq i \leq k}$ the vectors $\sum_{l=1}^{k}( \pm) C_{l} \mathrm{e}_{i}$, where the values of $C_{l}$ are described in Section 2. If it is found for some combination of \pm signs and $C_{l}$ 's and for some $e_{i,}$ that

$$
\left|\mathbf{e}_{i_{i}}\right|>\left|\sum_{l=1}^{k}( \pm) C_{l} \mathbf{e}_{i_{l}}\right|,
$$

the vector $\mathbf{e}_{i_{j}}$ is replaced by the vector $\mathbf{e}_{i_{i}}^{\prime}=\sum_{l=1}^{k}( \pm) C_{l} \mathbf{e}_{i_{l}}$ (the transformation from $E=\left(\mathrm{e}_{i}\right)_{1 \leqq i \leqq n}$ to $E^{\prime}=\left(\mathrm{e}_{1}, \mathrm{e}_{2} \cdots, \mathrm{e}_{i_{j}}^{\prime}, \cdots, \mathrm{e}_{n}\right)$ is unimodular $)$. Stage 1 of the 
algorithm is repeated on $E^{\prime}$. The algorithm must terminate after a finite number of steps, since if a basis is altered by an operation in stages 1 or 2 , the alteration consists in replacing a vector with integer coordinates with a shorter vector having integer coordinates.

Remark. In the initial consideration of the problem of finding reduced bases for $n>2$, the search technique suggested by Coveyou and MacPherson [3] was considered with some modifications suggested in van der Waerden [12]. Without preliminary reduction it was found in a typical example (of the type discussed in this paper) about $10^{19}$ vectors would have had to be examined to find the shortest nonzero vector. Techniques used in crystallography were also considered (see Azároff and Buerger [1] and Roof [10]). Tests showed that these techniques were unsatisfactory for our purposes, due to lack of precision and the amount of search required.

6. Finding Bases for Multiplicative Congruential Pseudo-Random Points. To apply the reduction algorithm to the determination of reduced bases for pseudorandom points of the form (3) or (5), it is necessary to find a basis of these points. The method of finding a basis is illustrated by an example.

Consider the example of the generator $(1)$ with $\lambda \equiv 5(\bmod 8)$ and $x_{0} \equiv 1(\bmod 4)$. To find a set of basis vectors for $G_{n}$ defined by (5), choose a set of $n+1$ vectors in $G_{n}$ as follows:

$$
\begin{aligned}
\mathrm{r}_{0} & =\left(1, \lambda, \lambda^{2}, \cdots, \lambda^{n-1}\right), \\
\mathrm{r}_{i} & =\left(4 \alpha_{i}+1, \lambda\left(4 \alpha_{i}+1\right)+h_{i}^{1} 2^{\beta}, \cdots, \lambda^{n-1}\left(4 \alpha_{i}+1\right)+h_{i}^{n-1} 2^{\beta}\right), \\
& i=1,2, \cdots, n,
\end{aligned}
$$

where $\alpha_{i}, h_{i}^{k}, i=1,2, \cdots, n, k=1,2, \cdots, n-1$, are arbitrary integers. A calculation gives

$$
\operatorname{det}\left(\mathbf{r}_{i}-\mathbf{r}_{0}\right)=2^{2+(n-1) \beta}\left|\begin{array}{cccc}
\alpha_{1} & h_{1}^{1} & \cdots & h_{1}^{n-1} \\
\alpha_{2} & \vdots & & \vdots \\
\vdots & \vdots & & \vdots \\
\alpha_{n} & h_{n}^{1} & & h_{n}^{n-1}
\end{array}\right|
$$

and $\left|\operatorname{det}\left(\mathbf{r}_{i}-\mathbf{r}_{0}\right)\right|$ has its minimum nonzero value when $\alpha_{1}=h_{2}^{1}=h_{3}^{2}=\cdots=$ $h_{n}^{n-1}=1$, the other determinant entries being zero. Thus a set of basis vectors of $G_{n}$ is given by (with these choices for $\alpha_{i}$ and $h_{\mathbf{i}}^{i}$ )

$$
\begin{aligned}
& \mathbf{r}_{1}-\mathbf{r}_{0}=4\left(1, \lambda, \lambda^{2}, \cdots, \lambda^{n-1}\right), \\
& \mathbf{r}_{i}-\mathbf{r}_{0}=\left(0,0, \cdots, 2^{\beta}, 0, \cdots, 0\right), \quad i=2,3, \cdots, n,
\end{aligned}
$$

where $2^{\beta}$ appears in the $i$ th place.

7. Examples. Tables 1 to 5 present a few examples. It is hoped that the captions are self-explanatory, except for "figure of merit" defined for a reduced basis $\left\{y_{i} ; i=1,2, \cdots, n\right\}$ by

$$
\text { figure of merit }=\frac{\min _{1 \leq i \leq n}\left|y_{i}\right|}{\max _{1 \leq i \leq n}\left|y_{i}\right|} .
$$




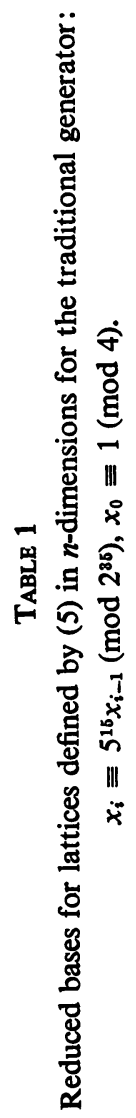

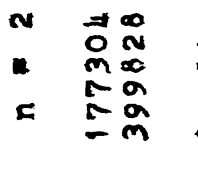
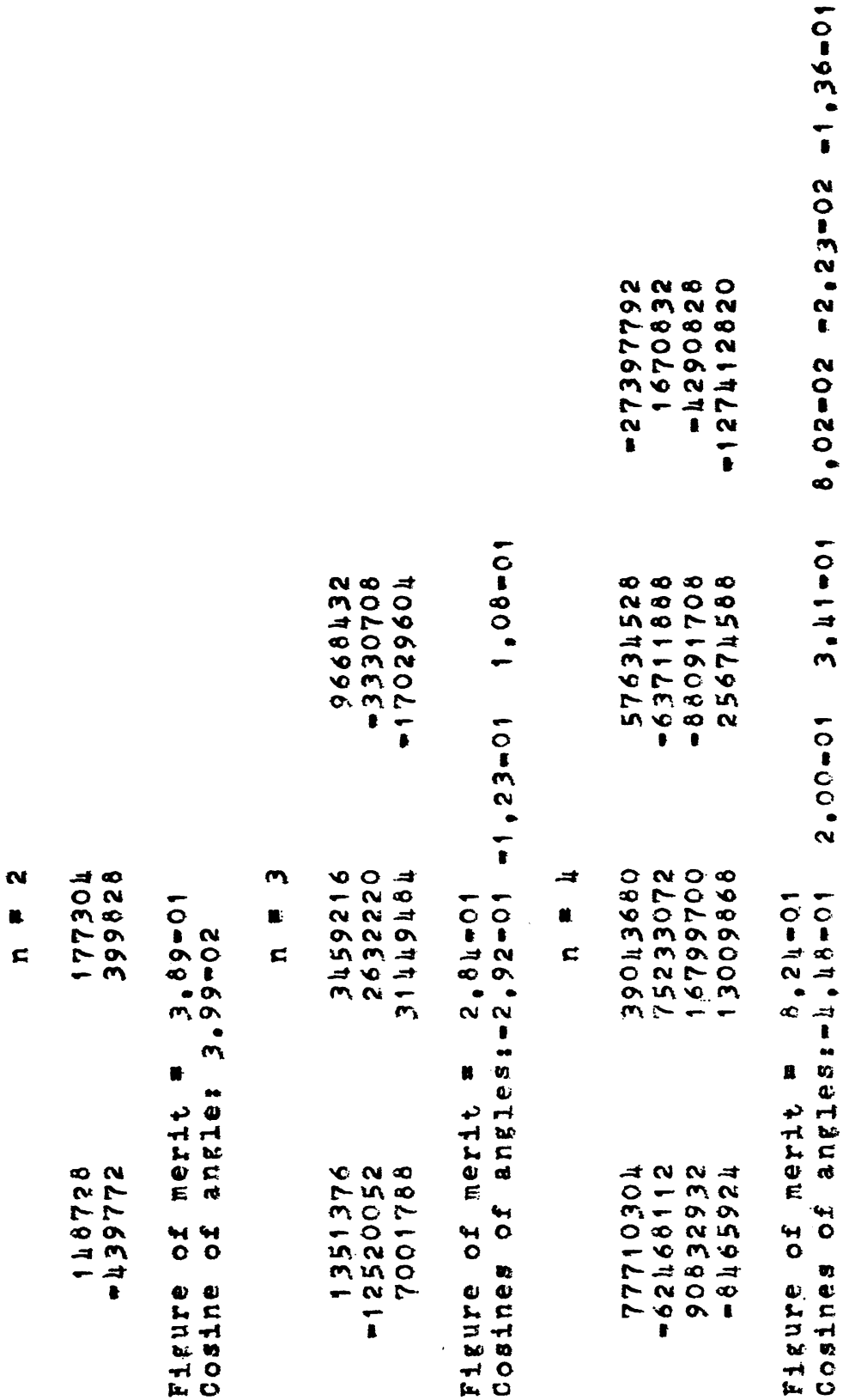


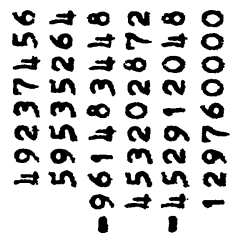

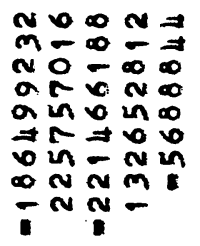

$00 \infty \Rightarrow 0$

$m \neq \infty \frac{0}{0}$ a $x \Rightarrow 0$ a $-1 \Rightarrow N \mathrm{~m}$ in a $\mathrm{mm}$ $\infty$ o 0 o $\infty$ $\infty \begin{array}{llll}\infty & \alpha & 0 & 0 \\ \sim & \alpha & \infty & -\infty\end{array}$ u $0=-\infty$ $\infty \div$

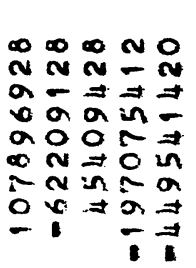

$0=000$ $-0 a-\infty$ c. $N-\Gamma+\infty$ rom on c $\quad 0-\Rightarrow 6 \Omega$ $x-\bar{a}$ in $-1-\infty=$ $m$ in $\rightarrow \rightarrow m$ N - ल

$0 \omega \neq 00$ 0 in 0 N $c m a \neq 0$ $r-v \equiv N$ $N$ in $N$ in $m$ - N uno in $N 000$ No $0 \mathrm{~m}=$ - $N$ i

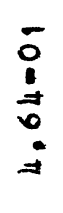

5
0
0
0
0
0
$=\infty$
$m-$
$m-$

$\div 0$

$\infty \neq$

$\Rightarrow 0$

mo

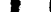

$\begin{array}{ll}0 & - \\ 0 & 0 \\ 0 & 0\end{array}$

$-0$

in -

$-\infty$

000

- 1

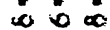

$\infty \Rightarrow$

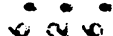

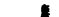

$=$

$0=0$

$+\pi$

S.

(1)

E.

4
0

$c$

a)

a)

$\Rightarrow$ 다

if 0
$0 \neq 000 \Rightarrow$

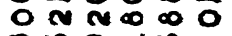
$\infty \circ 0=10-$ $=0 \Rightarrow$ in $0 \mathrm{~m}$ - in un un $\infty$ -amir $\Rightarrow 000$ in in mat $=$ in

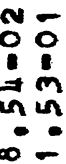

$\infty-$

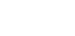

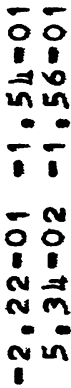

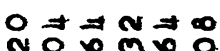

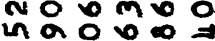
a o a 0 in N $00 \times$ N -00 a

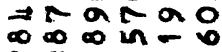
- $m \Rightarrow m \Rightarrow \infty$ $\Rightarrow$ un $m$ m . I

N-

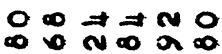
- $-\infty$ a in $N$ $\Rightarrow \Rightarrow 0 \times N-$ $\Rightarrow \nexists$ in $0 \mathrm{~m}$ in $0-0$ in $a \neq 0 \times 0$

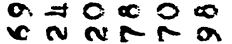
N N NrRa a - : $0 \Rightarrow 0$ un N $\Rightarrow$

- $\quad \Rightarrow 0 N \infty \neq 0$

- $\Rightarrow 0$ < $a=0$ a $0-\Rightarrow b 0$

c. $\quad \infty$ in in 6 in $\infty m \Rightarrow n=$ c. in $0=0-$ in

c. $=\frac{m}{m}=$

$--N-$

000

in 1

$m=0-$

- -

t Num

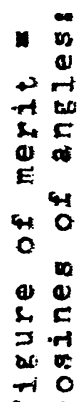

nov $\mathrm{d}=0$

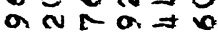

ur mar-

$a \neq-n-0$

$m-0 a v 0$

- mN Ln 0 in 0 io in OONaNR

$m=\Rightarrow=0 \div$

-io 


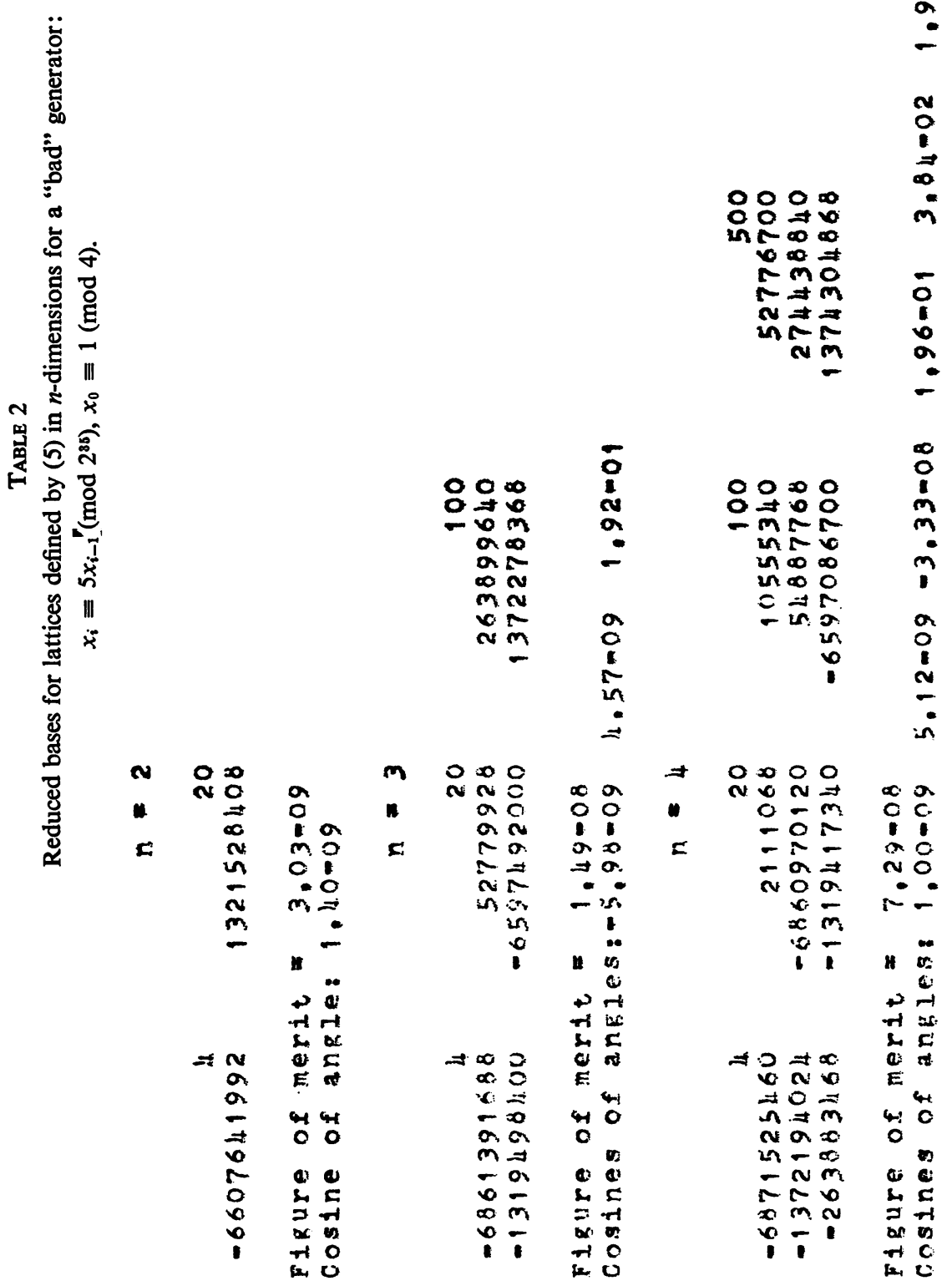




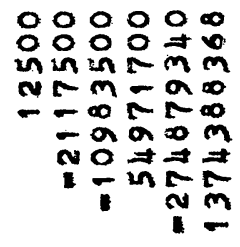

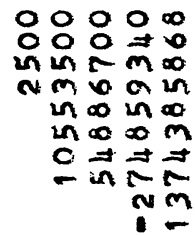

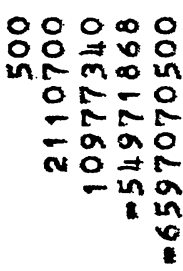

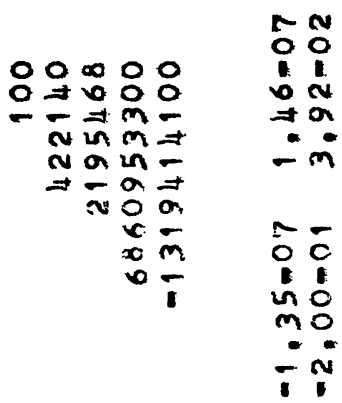

in 0 so 00

No $\infty$ o

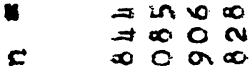

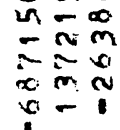

$\Rightarrow \infty \vee \alpha \Rightarrow$

$\infty-m v$

ar - in

mom?

$m \bigcirc m_{m}$

$a m \Rightarrow n$

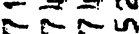

$\infty$ in $\sim$

$0-$ v
0
0
$m$

-
0
0
0
0

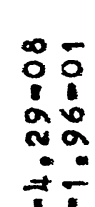

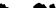

운

$\because \cdots$

둥

no

$-n$

r.m

000

$\Rightarrow$ a

c. 6

-

11

II $\begin{gathered}0 \\ 0 \\ 0 \\ 0\end{gathered}$

$\rightarrow$ be

Le 5

E

40

0

a.

c.

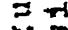

at os

in 0
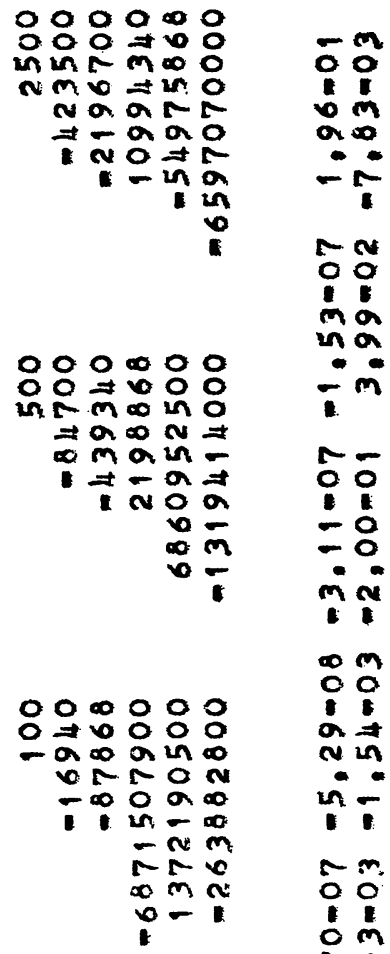

v

0 o0 00 Nल 0 \% 0

$m=-\infty$

mo $\mathrm{m}^{\infty}$ a $m=1$ $-\Rightarrow \rightarrow n$ $\sim r \pi$ in a $M N$ !

(c)

$0 \mathrm{~m}-$

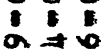

c in 0

in -

$\sim \cdots, \alpha$

000

15

$r \ln$

$\infty \approx m$

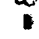

ON

${ }^{\circ} 0_{0} 0$

1.

N 0 \% 0

c una

- -1

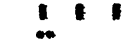

4

$\rightarrow+$

त) b

a $x$

$\Rightarrow 00 \times 0 \times$ U

o $\mathbf{N}-\boldsymbol{c}$

$0 . c \mathrm{~m} v \mathrm{~m}$

$060 \leq-1$

$=\infty$ i $\infty$ in

a $m \infty \infty$ in

$-\Rightarrow \Rightarrow \Rightarrow 0$

N N in -

c. $m$

a -1
$4-1$

0

a)

(5)

$2 \pi$

$\infty$

ar 0 

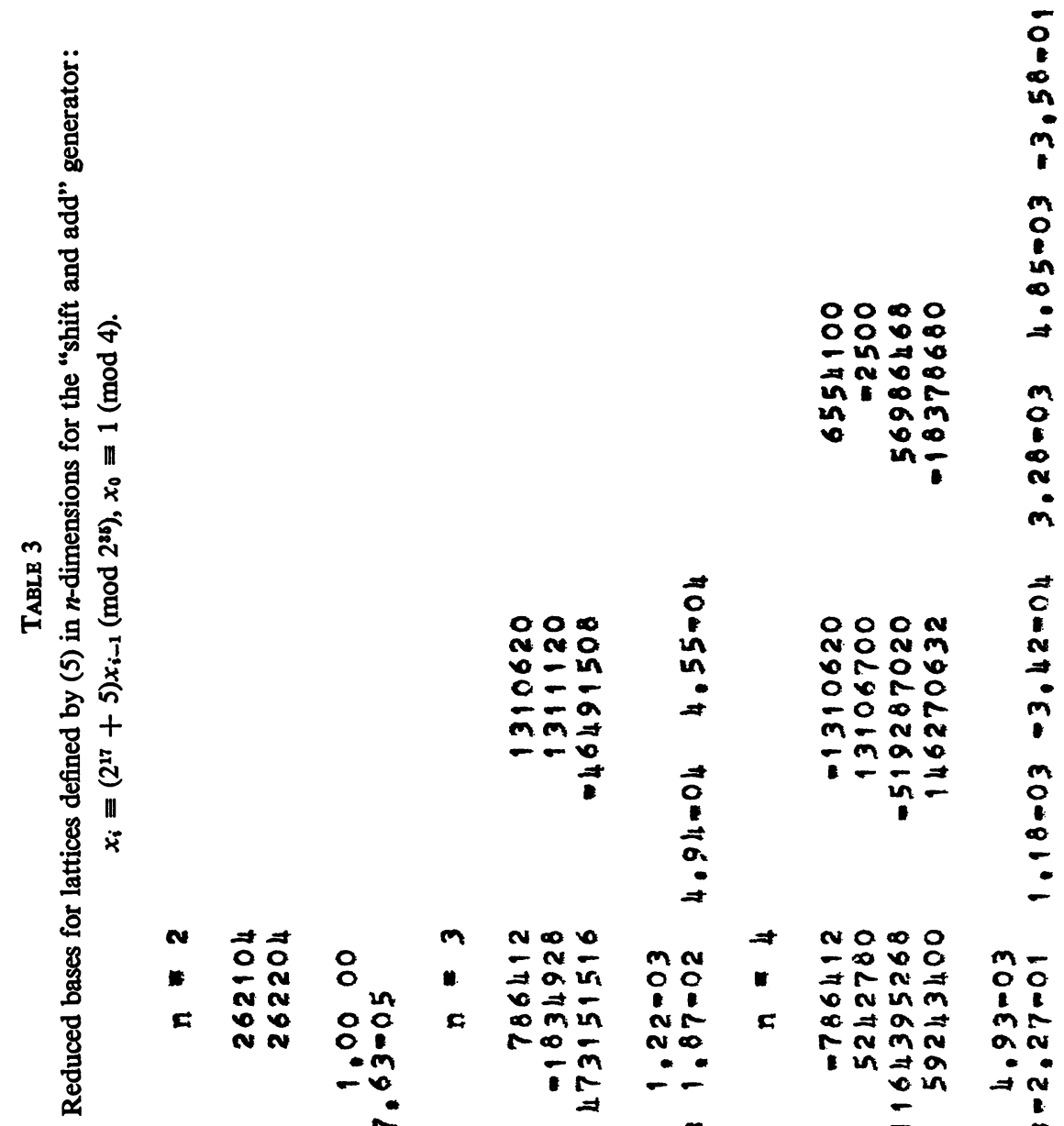

응

0

m

$\underset{\infty}{\infty} \div$

mo $0 \Rightarrow-$

N

$\div \frac{m}{m} \sim-$

-

$\div \div$

$\stackrel{+\infty}{+1}$

4 is

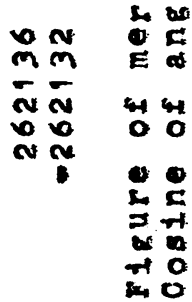

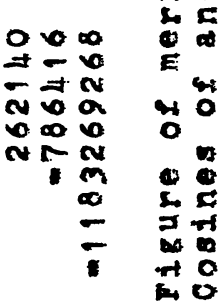

$000 \mathrm{~cm}$

중

-

$-0+\infty$

$m-\infty \pi$

$\overrightarrow{0}$
$\overrightarrow{0}$
$\ddot{0}$

- ma

n-

$m$
0
-

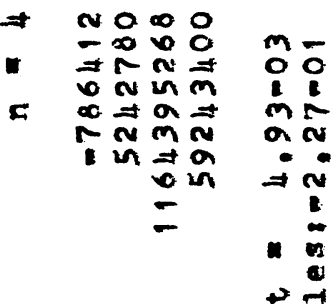

$\rightarrow$ bo

$0+\infty 0$ a

$\Rightarrow \exists \infty 0 \quad E$

तc a 0 म

cNOM

ain in un

- 00

$\infty$ in 1

Im 70

if 0 

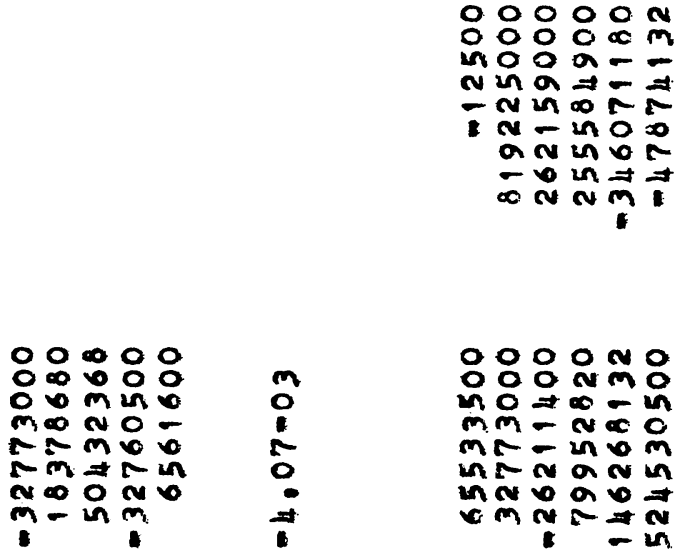

OOO 0 응 Motim $m m=\infty \neq c$ $m R=$ in $0 \mathrm{~m}$ in 2 a in

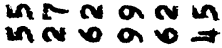

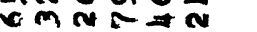

$m-$ in

$2 \pm$

=

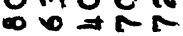
a o $0 \mathrm{mo}$ जR

o o a co

o. 0 N in

$-1-\Rightarrow$

in $\overrightarrow{1}$

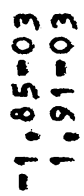

(a)

mo

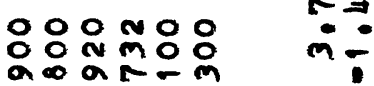

maOrON

- in - in una

voa 0 a

M에

- NNRー

$m$

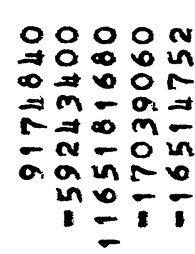

n-

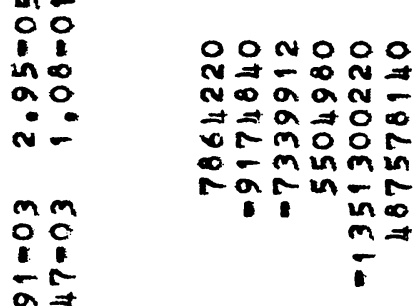

cor

11.

$-60$

$\ln \Rightarrow$

$\sim m$

$\rightarrow \infty+0$

- I mo

- $-m$ un m

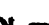

웅

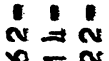

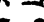

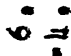

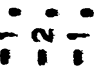
$n \Rightarrow 0 \infty 0 \times$
$\Rightarrow 0 N$
un $⿻ 00$
$m_{\infty \rightarrow 0}-0$
c $\infty$
- una
$\sim \circ \bullet \Rightarrow \Rightarrow$
in $\infty$
$m \rightarrow$

$N \neq \infty N \alpha \vec{v}$ Nm- man : $\infty 0-$ 0 in in in

- rmarga $0 \infty 00 \Rightarrow m$

$N \sim M m \infty$ min $\vec{x}$

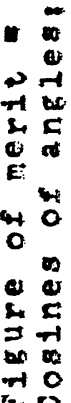
$-1$

$\Rightarrow \Rightarrow 0000$ $\infty \pi \infty \pm N 0$ $N \Rightarrow N \bar{m} v$ $\Rightarrow \overrightarrow{0}=m$ in $c \infty \Rightarrow \pm-\Rightarrow$ $n \pi \infty m \infty$

- mama $r=6 m$ mu-

Noto

- 1. an un $00 \sim m$ $\Rightarrow n-m$
- $+4$
if 2
E
40 0
0
c)
3 of 0

in 0 


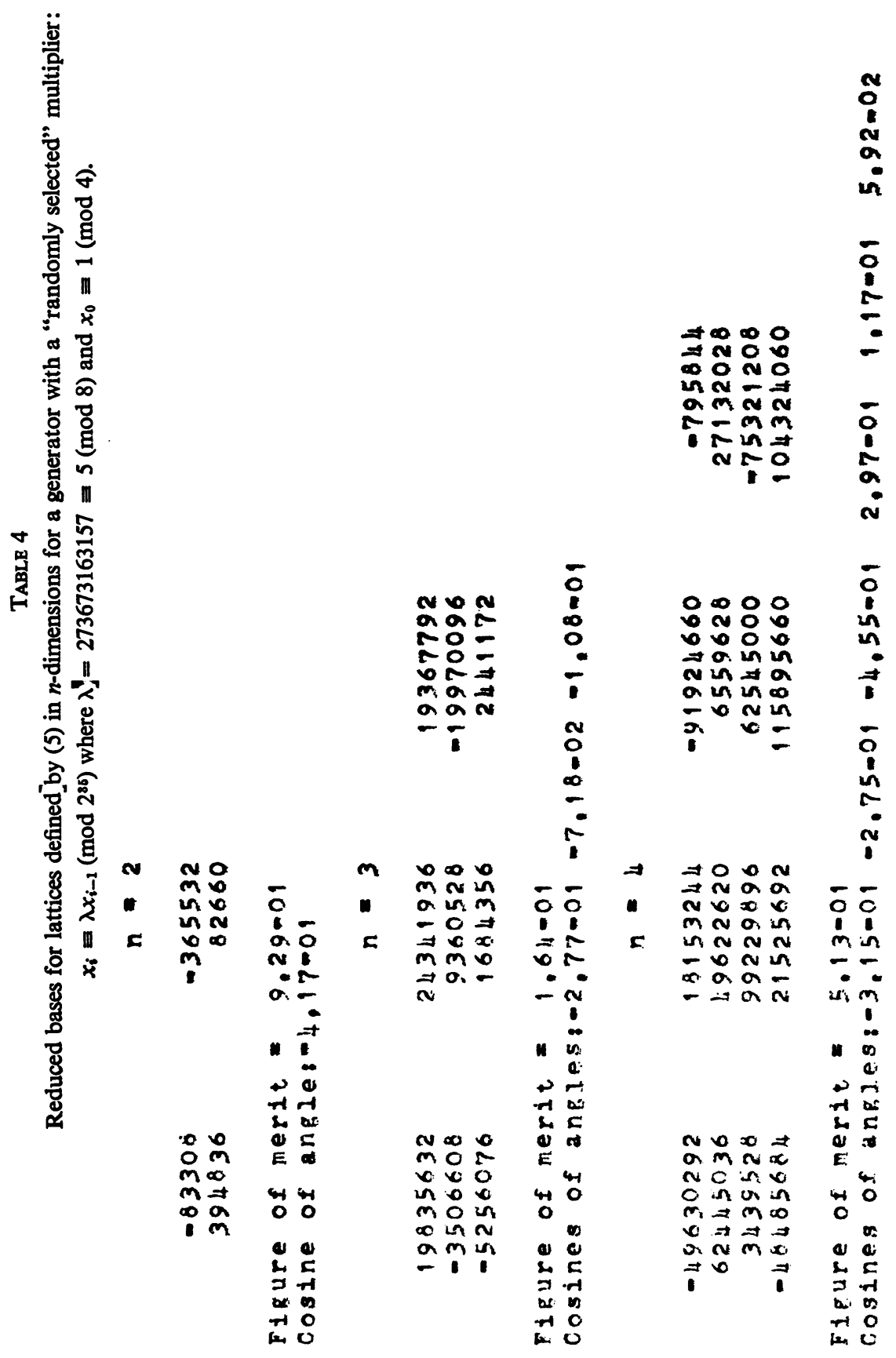




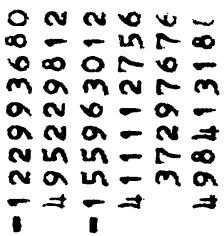

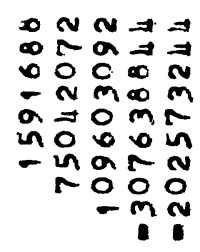

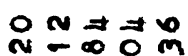
$m_{i \infty}^{\infty} \rightarrow$

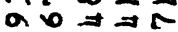
$\Rightarrow$ r a n $\infty$ in $-a$ in mo 0 o. 0 $\infty$ in $0<\Rightarrow$ $-1-2$

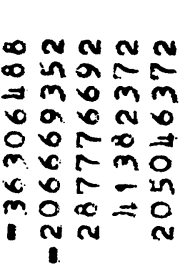

- $N=0$

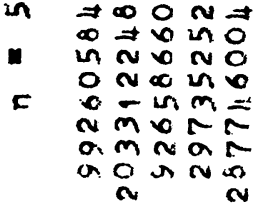

$0 \times .0$. - - r w e c $n_{1}<0 \Rightarrow$ - $0.00 \bar{c}$ $\Rightarrow 0$ N mo on $N$ - min $\alpha 0$ $\Rightarrow$ un un

N $-\infty=$

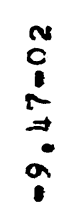

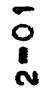

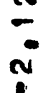

-0
00
$0=$
$0=$
0

둥

1 i

0 in

0 -

$\omega \Rightarrow$

-

50

:

ㄴ. $\Rightarrow$

$-n$

- 0

O

co in

$\infty a$

:-

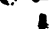

$1 \%$

$\rightarrow+4$

+1 ax

I.

a.

5

40

0

a)

2.

$=-4$

+10
0
- NO 0 D N T- 0 - 0 in - $m$ un 0 N - o un a $\Rightarrow m \sim 0 \Rightarrow N$ un in o N $\mathrm{N}$ nก $\sim m$ in in MNOara N - o in in

$\sim \infty 0 \sim 0 N$ $-\Rightarrow N-\infty N$ - $-\infty-a-$ $\alpha \Rightarrow N \infty N-$ $\Rightarrow \neq 0$ in in in त 0 - 0 i vo 0 in $-n \rightarrow-0-$
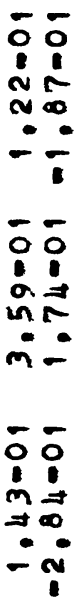

- O N $00 \infty$ $00 m-0 \Rightarrow$ - a mar = c N $0 \Rightarrow \exists \exists$ ON $-7=$ in 0 우욤 in - ma 0

$m$ in $=0$

- N 0 NO

- $\quad \infty w 0$ in rm $m$ r a H. - in $a m \infty$ - co a a a c $\therefore-\infty<N-$ ar:

$\overrightarrow{\overrightarrow{1}} \overrightarrow{\mathrm{i}}-\mathrm{in}$ in $\rightarrow N x \rightarrow \infty x$ - $A-m a n$ $\infty \infty \Rightarrow \pm-\infty$ $c \Rightarrow v 0$ in in c v - $\Rightarrow$ a 0 o. $m m_{1} c \rightarrow-$ - $v$ in 0 a

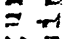
of 0

\section{$-\sigma-$}

웅응 ina r 10 $0 m-m$

12 $+\stackrel{4}{a}$ - 4 is 2. 5 E: 4 0 is a - 1 


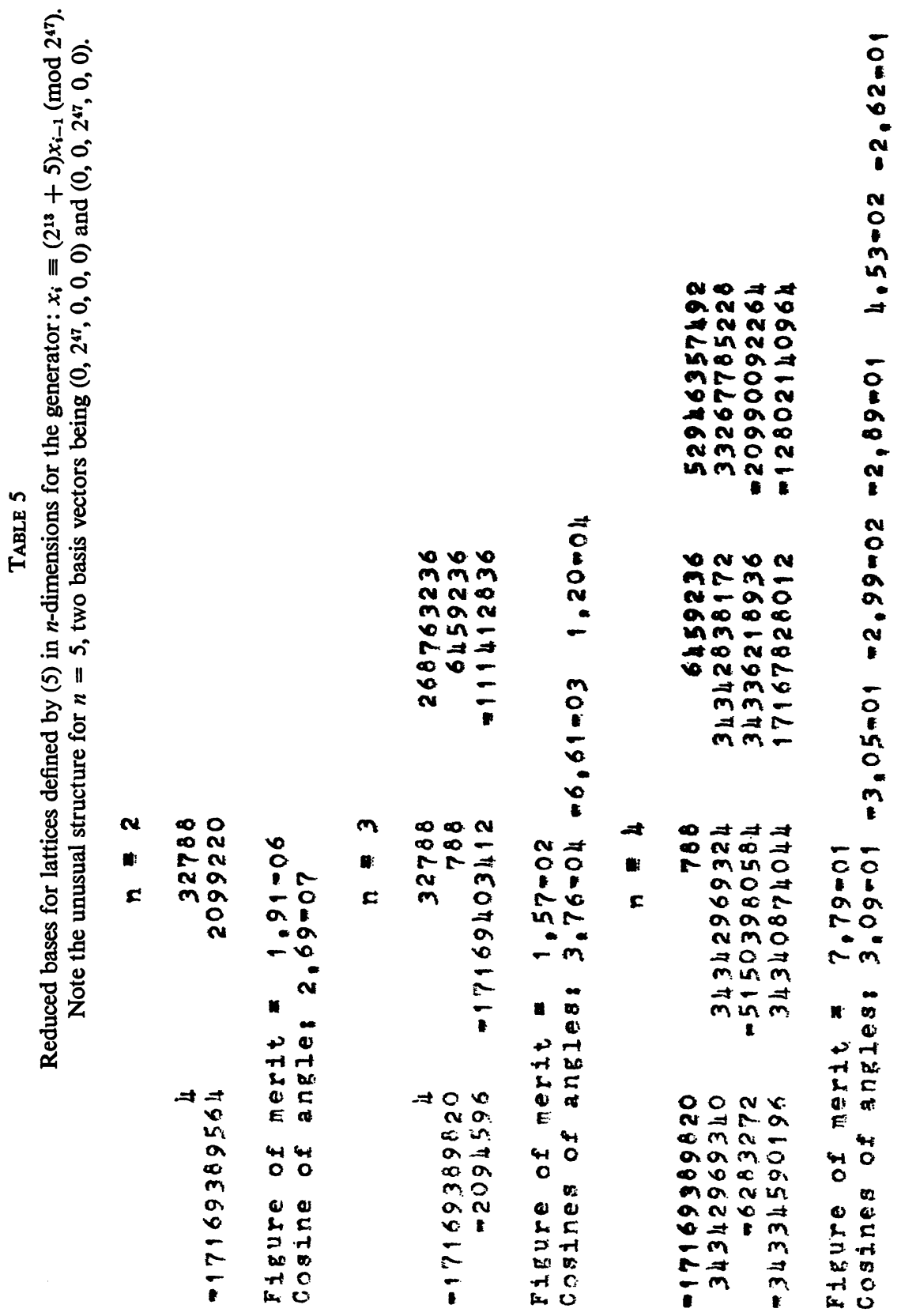




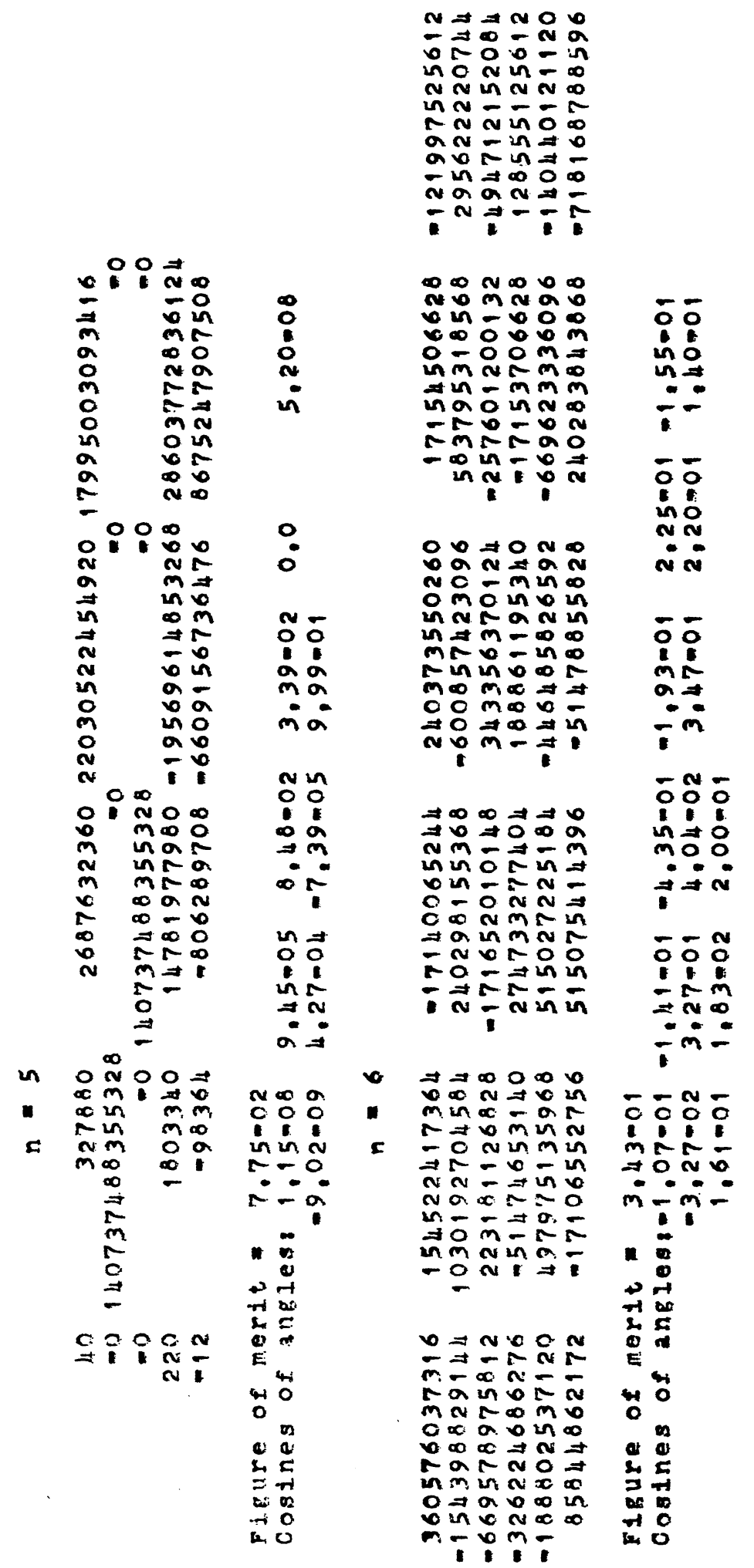


The figure of merit provides some measure (neglecting angle) of the departure of the reduced cell from "squareness." $x \cdot x x-0 a$ means $x \cdot x x \cdot 10^{-a}$. The angles refer to angles between the edges. Angles between higher-dimensional flats have not been calculated, but it might be useful to do so. Each row of the tables lists the components of a vector.

It is seen from these tables that multipliers of simple structure, such as 5 or $2^{17}+5$ produce lattices of points which depart greatly from a uniform distribution throughout the cube. Multipliers of more complex structure, such as $5^{15}$ or the "randomly" selected multiplier 273673163157 produce better lattices. A typical time for the computation of a table on Maniac II was 20 minutes. The CDC 6600 is perhaps 8 times faster than Maniac II.

It is hoped that more examples with a more complete discussion can be presented in the future.

8. Connection Between Lattices of Pseudo-Random Points and the Theory of Discrepancy. Zaremba [15], Schmidt [11], and others (see references in [11] and [15]) have discussed the notion of the discrepancy $D(S)$ of a finite number of points $S$ in the unit cube $I_{n} \subset E^{n}$. The quantity $D(S)$ can be used to estimate the error in the evaluation of a multidimensional integral. As an example, if $f(x, y)$ is of bounded variation in the sense of Hardy and Krause over $\bar{I}_{2}$ and $S=\left\langle x_{0}, \cdots, x_{N-1}\right\rangle$ is an arbitrary sequence of points in $I_{2}$, then

$$
\left|\int_{I_{2}} f(\mathbf{x}) d \mathbf{x}-N^{-1} \sum_{k=0}^{N-1} f\left(\mathbf{x}_{k}\right)\right| \leqq V^{2}(f) D(S)+V(f(x, 1)) D(X)+V(f(1, y)) D(Y),
$$

where $V$ and $V^{2}$ denote one- and two-dimensional variation and $X$ and $Y$ are projections of $S$ on the $x$ and $y$ axis respectively. Roth (see [15]) has proved that, for $E^{n}$,

$$
D(S) \geqq C_{n} N^{-1}(\log N)^{(n-1) / 2}
$$

for some constant $C_{n}$.

It seems to be a reasonable conjecture that if the figure of merit (see Section 7) of a lattice $G_{n}$ is very small, then $D\left(G_{n} \cap I_{n}\right)$ is large. Conversely, if the figure of merit is near $1, D\left(G_{n} \cap I_{n}\right)$ is small.

It should be remarked that the application of this lattice theory to much shorter segments of the full period of the generator sequence depends on the extent to which the lattice properties are reflected in the segments.

Acknowledgment. The authors thank Dr. W. W. Wood of this laboratory for valuable help and suggestions in the preparation of this paper.

Postscript. After completion of the above paper, the following important paper came to the authors' attention:

R. R. Coveyou, "Random number generation is too important to be left to chance," Studies in Appl. Math., v. 3, 1970, pp. 70-111.

That paper has things in common with our paper. However, our paper was written independently and differs from the former in important details. It was thought best to not revise the present paper. 
University of California

Los Alamos Scientific Laboratory

Los Alamos, New Mexico 87544

1. L. V. AzÁroff \& M. J. Buerger, The Powder Method in X-Ray Crystallography, McGraw-Hill, New York, 1958.

2. J. W. S. CASSELS, An Introduction to the Geometry of Numbers, Die Grundlehren der math. Wissenschaften in Einzeldarstellungen mit besonderer Berücksichtigung der Anwendungsgebiete, Band 99, Springer-Verlag, Berlin, 1959. MR 28 \#1175.

3. R. R. COVEYOU \& R. D. MACPHERSON, "Fourier analysis of uniform random number generators," J. Assoc. Comput. Mach., v. 14, 1967, pp. 100-119. MR 36 \#479.

4. J. N. FranKLIN, "Deterministic simulation of random processes," Math. Comp., v. 17, 1963, pp. 28-59. MR 26 \#7125.

5. J. N. FRANKLIN, "Equidistribution of matrix-power residues modulo 1," Math. Comp., v. 18,1964 , pp. $560-568$.

6. S. W. Golomb, L. D. Baumert, M. F. Easterling, J. J. StifFler \& A. J. Viterbi, Digital Communications with Space Applications, Prentice-Hall, Englewood Cliffs, N. J., 1964. $36 \# 7297$.

7. B. JANSSON, Random Number Generators, Almquist \& Wiksell, Stockholm, 1966. MR

8. G. Marsaglia, "Random numbers fall mainly in the planes," Proc. Nat. Acad. Sci. U.S.A., v. 61, 1968, pp. 25-28. MR 38 \#3998.

9. H. MinkowSKI, "Zur Theorie der positiven quadratischen Formen," J. Reine Angew. Math., v. 101, 1887, pp. 196-202.

10. R. B. Roof, JR., A Theoretical Extension of the Reduced-Cell Concept in Crystallography, Los Alamos Scientific Laboratory Report, LA-4038, 1969.

11. W. SCHMIDT, "Irregularities of distribution. IV," Invent. Math., v. 7, 1969, pp. 55-82. MR 39 \#6838.

12. B. L. VAN DER WAERDEN, "Die Reduktionstheorie der positiven quadratischen Formen," Acta Math., v. 96, 1956, pp. 265-309. MR 18, 562.

13. P. H. VERDIER, "Relations within sequences of congruential pseudo-random numbers," J. Res. Nat. Bur. Standards Sect. B, v. 73B, 1969, pp. 41-44. MR 39 \#1081.

14. W. W. Wood, "Monte Carlo calculations for hard disks in the isothermal-isobaric ensemble,"J. Chem. Phys., v. 48, 1968, pp. 415-434.

15. S. K. 7AREMBA. "The mathematical basis of Monte Carlo and quasi-Monte Carlo methods," SIAM Rev., v. 10, 1968, pp. 303-314. MR 38 \#1810. 